\title{
Embolismo pulmonar tumoral: Reporte de dos casos
}

\author{
Felipe J Chertcoff 1,2 , Nicholas C Emery $y^{1,4}$, Renzo Villagomez ${ }^{1}$, \\ Miguel A Veltri ${ }^{2}$, Julio Venditti ${ }^{3}$, Pablo Young ${ }^{4}$, Jorge Ubaldini ${ }^{2}$. \\ Pulmonary tumor embolism: \\ Report of two cases
}

Dyspnea and hypoxemia in a patient with cancer may have several causes, including infections, thromboembolism, metastases, and pulmonary injuries by drugs. We report a 47 year-old female with breast cancer and a 70 year-old male with urinary bladder cancer who were admitted for dyspnea, hypoxemia and pulmonary hypertension. Chest X rays and CT scans were normal. The ventilation-perfusion scintigram was highly suspicions of thromboembolism in the female. The male died 16 hours after admission and the post mortem examination revealed the presence of tumor cells in the pulmonary vasculature (Rev Méd Chile 2009; 137: 1613-16).

(Key words: Breast neoplasms; Intracraneal embolism and thrombosis; Urinary bladder neoplasms)

Recibido el 30 de marzo, 2009. Aceptado el 6 de octubre, 2009.

${ }^{1}$ Servicios de Neumonología, ${ }^{2}$ Terapia Intensiva, ${ }^{3}$ Anatomía Patológica y ${ }^{4}$ Clínica Médica, Hospital Británico de Buenos Aires. Argentina.

$E^{1}$ embolismo tumoral pulmonar se produce cuando células tumorales impactan en el lecho vascular pulmonar. Puede comprometerlo en distintas maneras: émbolos tumorales grandes y proximales, émbolos pequeños en la microvasculatura, diseminación linfática, o una combinación de ambos.

Es un cuadro de difícil diagnóstico, que exige un alto índice de sospecha, las manifestaciones clínicas son pocas e incluyen: disnea progresiva, hipoxemia, hipertensión pulmonar o corazón pulmonar agudo en un paciente con estudios radiográficos normales. En muchas ocasiones el diagnóstico es postmortem.

Comunicamos dos casos que exigieron el diagnóstico diferencial con el tromboembolismo trombótico también frecuente en pacientes con cáncer.

Correspondencia a: Dr. Pablo Young. Hospital Británico. Perdriel 74 (1280) Buenos Aires, Argentina. Tel: 541143096400. Fax: 5411 43043393. E mail: pabloyoung2003@yahoo.com.ar
Caso 1. Mujer de 47 años, con antecedentes de cáncer de mama tratado con mastectomía total y vaciamiento axilar (adenocarcinoma ductal infiltrante N2M2G2) diagnosticado 10 años antes. Consultó por disnea progresiva hasta hacerse de reposo y astenia de un mes de evolución. Al examen físico presentaba taquipnea como único hallazgo. Su saturación arterial de $\mathrm{O} 2$ ( $\mathrm{SaO} 2)$ fue de 93\% sentada y $88 \%$ en decúbito dorsal. El laboratorio evidenció hematocrito de $31 \%$ (HTO) (poiquilocistosis, algunos esquizocitos), glóbulos blancos $4.800 \times \mathrm{xm}^{3}$, plaquetas $41.000 \times \mathrm{mm}^{3}$, LDH $1569 \mathrm{U} / \mathrm{L}$, eritrosedimentación $30 \mathrm{~mm} / 1 \mathrm{~h}$, dímero D $1.432 \mathrm{ug} / \mathrm{ml}(\mathrm{VN}$ : $\varangle 0,5)$, gases arteriales $\mathrm{pH} 7,42, \mathrm{PaCO} 232 \mathrm{mmHg}$, $\mathrm{PaO} 252 \mathrm{~mm} \mathrm{Hg}, \mathrm{EB}+3$ (FIO2: 21\%). Una radiografía de tórax fue normal. El eco Doppler de miembros inferiores (MMI) no evidenció trombosis venosa profunda (TVP), el cintigrama V/Q pulmonar mostró alta probabilidad de embolismo pulmonar (TEP) con defecto de perfusión subsegmentario bilateral (Figura 1). La tomografía computada helicoidal (TACH) de tórax con protocolo para TEP fue negativa. Se 
Figura 1. Cintigrama de ventilación/perfusión con el patrón típico de embolismo segmentario marginal. El tamaño de la imagen pulmonar por perfusión es menor que la ventilación por oclusión vascular periférica (flechas).

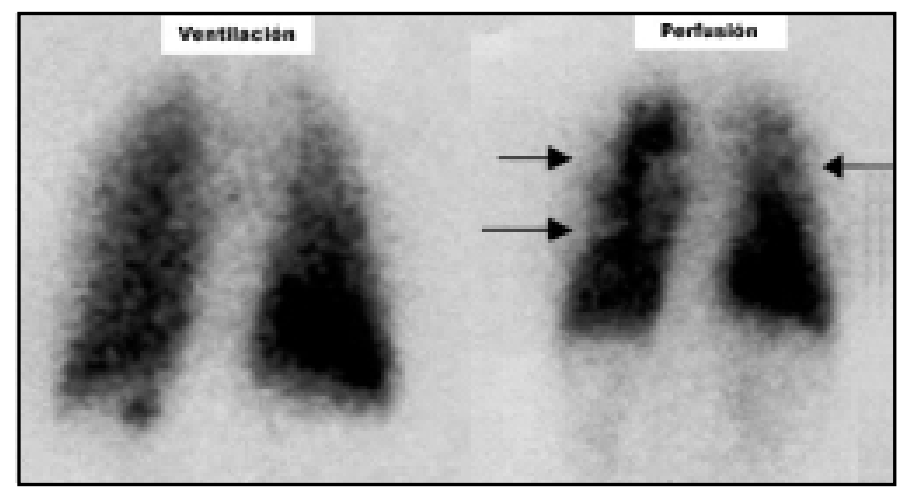

realizó un ecocardiograma 2D que mostró ventrículo derecho (VD) límite superior normal, función sistólica del ventrículo izquierdo (FSVI) conservada, insuficiencia tricuspídea (IT) moderada con presión sistólica pulmonar (PSAP) de $63 \mathrm{mmHg}$ y la arteriografía pulmonar fue normal. El catéter de Swan Ganz mostró presión arterial pulmonar (PAP) de 59/25 $\mathrm{mmHg}$, presión media pulmonar (PMAP) de 35 $\mathrm{mmHg}$, resistencia vascular pulmonar (RVP): 400 dinas $\mathrm{x}$ seg $\mathrm{x} \mathrm{cm}^{-5} \mathrm{y}$ el frotis de sangre venosa mixta enclavada fue negativo para células neoplásicas. Ante la persistencia de los síntomas y la tricitopenia marcada se realizó punción de médula ósea donde se observa una médula hipocelular y acúmulos celulares atípicos tipo "colgajos" que corresponden a secundarismo tumoral por adenocarcinoma de origen mamario.

Caso 2. Varón de 70 años, con antecedentes de carcinoma indiferenciado de vejiga y adenocarcinoma de próstata tratado con cistoprostatectomía radical 2 años antes.

Cinco meses previos a su última intemación por distensión abdominal y ascitis se efectuó una TACH de abdomen y pelvis que mostró extensa alteración de planos grasos mesenténicos con ganglios aumentados de tamaño. Líquido libre perihepático, periesplénico, interasas y en ambos espacios parietocólicos, adenopatías inguinales bilaterales con probable carcinomatosis penitoneal. Inició quimioterapia. Ingresó por disnea clase funcional III y dolor abdominal de 3 días de evolución. El laboratorio mostró glóbulos blancos de $6.300 \mathrm{x} \mathrm{mm}^{3}$, HTO 35\%, troponina T 0,011 ng/ml (VN: $\varangle 0,01$ ), gases en sangre $\mathrm{pH} 7,50, \mathrm{PaO} 252$ mmHg, PaCO2 26 mmHg, EB -2, SaO2 85,9\% (FIO2 0,35). Eco Doppler de MMI sin TVP. La radiografía de tórax fue normal. Se efectuó TACH de tórax con protocolo para TEP donde no se observan defectos de relleno hasta vasos de $4^{\text {a }}$ generación, sin adenopatías, una banda de hipoventilación basal izquierda y opacidades parenquimatosas en lóbulo inferior derecho con líneas subpleurales aisladas sin signos de linfangitis. Evolucionó con disnea clase funcional IV, taquipnea y cianosis. SaO2: 91\% con máscara con reservorio (FIO2: 100\%), mala mecánica respiratoria, por lo que fue intubado y conectado a asistencia respiratoria mecánica. Un ecocardiograma Doppler evidenció leve dilatación del VD, resto de las cavidades normales, insuficiencia tricuspídea moderada, hipertensión pulmonar sistólica de $78 \mathrm{mmHg}$. Se colocó catéter de Swan-Ganz que mostró PAP 48/20, PMAP 29 mmHg, PW: 8 mmHg, RVP 352 dinas x seg $\mathrm{x} \mathrm{cm}^{-5}$. A las 16 horas de su ingreso el paciente fallece. La biopsia pulmonar postmortem mostró colgajos de células atípicas en el lecho vascular pulmonar compatibles con carcinoma (Figura 2).

\section{DisCUSIÓN}

La embolia tumoral es considerada una entidad clínica infrecuente, su incidencia en autopsias ha sido descrita entre $3 \%$ y $26 \%^{1-3}$ de los pacientes con tumores sólidos, más frecuentemente de mama, estómago y pulmón, pero estudios retrospectivos mostraron que sólo $8 \%$ de la morbilidad o mortalidad se relacionó con esta patología ${ }^{3-6}$.

Otros tumores que han sido descritos como causantes de embolia tumoral pulmonar son, a saber: hígado, próstata, páncreas, ovario, vejiga, colorrectal, riñón, mesotelioma, tumor de Wilms ${ }^{1}$. 


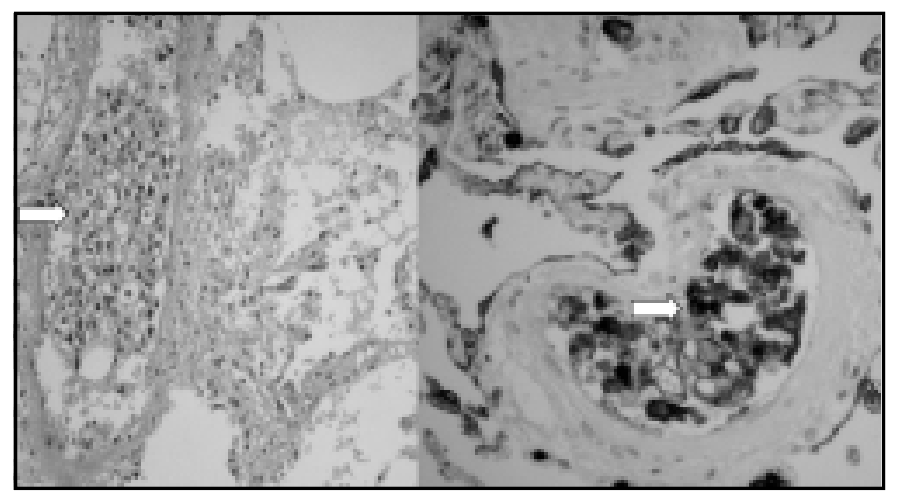

Figura 2. Biopsia pulmonar (postmortem). A la izquierda: vaso sanguíneo con grupo de células atípicas de estirpe epitelial junto con glóbulos rojos. Derecha: con mayor aumento $(20 \mathrm{x})$ vaso con células con marcación positiva para citoqueratinas AE1- AE3.

La presentación clínica refleja la progresiva oclusión del lecho vascular pulmonar con disnea de evolución aguda o subaguda descrita entre $57 \%$ y $100 \%$ de los pacientes. Los mismos pueden presentar además tos, dolor abdominal o torácico como signos inespecíficos de sobrecarga derecha. Los signos físicos de insuficiencia cardiaca derecha sólo han sido reportados en $15 \%$ a $20 \%$ de los $\operatorname{casos}^{1-3}$. La presencia de disnea en un paciente con cáncer en general orienta hacia existencia de infección, efecto colateral de drogas u otras manifestaciones del tumor primitivo ${ }^{7}$, pero dado el mecanismo fisiopatológico común y la frecuencia del mismo el tromboembolismo trombótico es el principal diagnóstico diferencial a tener en cuenta como ocurrió en nuestros dos enfermos.

La hipoxemia con radiología normal y un ecocardiograma que revela signos de hipertensión pulmonar o sobrecarga ventricular derecha hacen sospechar el tromboembolismo trombótico. Este es dos veces más frecuente en pacientes con cáncer, los cuales son además añosos, con comorbilidades, inmovilización y frecuentes estados de hipercoagulabilidad $^{8}$. La negatividad de los estudios orientados a su búsqueda, como en nuestros casos, permiten dirigir el diagnóstico hacia otro tipo de embolismo.

La radiología permite descartar linfangitis y enfermedad intersticial, aunque pueden coexistir, en muchos casos. En la tomografía computada fue descrito un patrón inespecífico subsegmentario de dilatación arterial pulmonar en estos pacientes, aunque lo más frecuente, como observamos en nuestros pacientes es la ausencia de signos de enfermedad parenquimatosa pulmonar, linfática 0 vascular.
El cintigrama de ventilación-perfusión tiene una utilidad diagnóstica mayor como se ve en la primer paciente; aparecen defectos subsegmentarios periféricos múltiples de perfusión con ventilación normal con lo que se ha denominado modelo de contomo segmentario (segmental contour pattern) que ha sido descrito como específico de los pacientes con enfermedad microvascular aunque otras patologías como las vasculitis, las embolias sépticas y la hipertensión pulmonar primaria pueden darlo $0^{9,10}$.

Sobre los pacientes con embolismo tumoral la arteriografía pulmonar no es sensible ni específica y en sólo 2 de 15 enfermos estudiados se demostró retraso e interrupción del flujo en arterias de tercero a quinto orden ${ }^{11}$. La arteriografía pulmonar utilizada en el primer caso fue realizada dada la alta probabilidad clínica de embolia de la paciente y el hecho de carecer de tomógrafo multislice que incrementa las posibilidades del diagnóstico de embolia de pulmón trombótica en este grupo de pacientes.

El cateterismo derecho con colocación de catéter de Swan-Ganz nos ayudó a confirmar, cuantificar o descartar hipertensión pulmonar como ocurrió en nuestros pacientes, ambos con hipertensión leve (sobreestimada por la ecocardiografía) y permite la obtención de sangre capilar enclavada para su análisis citológico que ha sido descrita como útil para el diagnóstico de microembolismo tumoral y linfangitis carcinomatosa. Sobre este método los autores recomiendan tener en cuenta que en algunos casos la citología vascular puede "contaminarse" con células malignas procedentes del hígado si el catéter no está enclavado y algunos falsos positivos han sido reportados debido a la presencia abundante de megacariocitos que podían confundirse. Su 
negatividad por supuesto no excluye la presencia de esta patología 12,13 .

La biopsia transbronquial puede permitir el diagnóstico, pero la biopsia de pulmón a cielo abierto o por videotoracoscopia es el método definitivo aunque la insuficiencia respiratoria y la inestabilidad hemodinámica limitan su utilización ${ }^{14}$.

Los patrones anatomopatológicos descritos son varios, a saber: 1) embolia tumoral con tromboendarteritis y anemia hemolítica microangiopática (probable en nuestra primer paciente); 2) embolia tumoral pura con manojos de células tumorales (nuestro segundo caso); 3) embolia tumoral con endarteritis fibroproliferativa; y 4) linfangitis con compresión arterial ${ }^{15,16}$.

El diagnóstico fue en el primer caso de exclusión en una paciente con antecedentes de

\section{REFERENCIAS}

1. Roberts Ke, Hamele-Bena D, Sadi A, Stein CA, Cole RP. Pulmonary tumor embolism: A review of the literature. Am J Med 2003; 115: 228-32.

2. Seppata N, Cala A, Kiebe S. Unusual presentation of pulmonary tumor thrombotic microangiopathy with no detectable primary tumor. J Postgrad Med 2009; 55: 38-40.

3. Winterbauer RH, EIFenbein IB, BaLL WC JR. Incidence and clinical significance of tumor embolization to the lung. Am J Med 1968; 45: 271-80.

4. MARINI JJ, BILNOSKI W, HuSEBY JS. Acute cor pulmonale resulting from tumor microembolism. West J Med 1980; 132: 77-88.

5. Kridel R, Myit S, Pache JC, Gaspoz JM. Pulmonary tumor embolism: a rare cause of acute right heart failure with elevated D-dimers. J Thorac Oncol 2008; 3: 1482-3.

6. Keenan NG, Nicholson AG, Oldershaw PJ. Fatal Acute pulmonary hypertension caused by pulmonary tumour thrombotic microangiopathy. Int J Cardiol 2008; 124: 11-3.

7. Case Records of the Massachussets General Hospital (Case 19-1995), N Engl J Med 1995; 332: 1700-07.

8. Piazza G, Goldhaber SZ. Acute Pulmonary Embolism Part I: Epidemioloy and Diagnosis. Circulation 2006; 114: e28-e32. cáncer de mama, hipertensión pulmonar leve, hipoxemia con ausencia de patología pulmonar radiológica, anemia hemolítica microangiopática, invasión de médula ósea por un tumor diseminado, cintigrama de alta probabilidad con patrón característico y arteriografía negativa, y confirmado por la biopsia postmortem en el segundo caso.

Como conclusión, el microembolismo tumoral es una complicación muy poco frecuente y diagnosticada postmortem. Debe ser sospechada en pacientes con antecedentes de cáncer y disnea aguda 0 subaguda con o sin hipertensión pulmonar o sospecha de embolia donde no se puede confirmar su origen trombótico. La suma de la sospecha clínica más un cintigrama pulmonar característico pueden orientar y el mismo si bien puede ser confirmado por citología vascular o biopsia de pulmón, su diagnóstico a veces es sólo de exclusión.

9. Moores LK, Burrell LM, Morse RW, Belgrave CH, BaLingit AG. Diffuse tumor microembolism a rare cause of a high-probability perfusion scan. Chest 1997; 111: 1122-5.

10. CRANe R, Rudd TG, Dail D. Tumor microembolism: pulmonary perfusion pattern. J Nucl Med 1984; 25: 877-80.

11. Schriner RW, Ryu JH, Edwards WD. Microscopic pulmonary tumor embolism causing subacute cor pulmonale: a difficult antemortem diagnosis. Mayo Clin Proc 1991; 66: 143-8.

12. MASON RG, Ruggieri J. Pulmonary microvascular citology. A new diagnostic application of the pulmonary artery catheter. Chest 1985; 88: 908-14.

13. Babar SI, Sobonya RE, SNyder LS. Pulmonary microvascular citology for the diagnosis of pulmonary tumor embolism. West J Med 1998; 168: 47-50.

14. Nakamura H, Adachi H, Sudoh A, Yagyu H, Kishi K, $\mathrm{OH}$-IsHi S ET AL. Subacute cor pulmonale due to tumor embolism. Intern Med 2004; 43: 420-3.

15. Von Herbay A, Illes A, Waldherr R, Otto HF. Pulmonary tumor thrombotic microangiopathy with pulmonary hypertension. Cancer 1990; 66: 587-92.

16. PINCKARD JK, WiCK MR. Tumor-related thrombotic pulmonary microangiopathy: Review of pathologic findings and pathophysiologic mechanisms. Ann Diagn Pathol 2000; 4: 154-7. 